\title{
Estudo da difusão da tecnologia móvel celular no Brasil: uma abordagem com o uso de Dinâmica de Sistemas
}

\author{
Júlio César Bastos de Figueiredo ESPM
}

\section{RESUMO}

Este trabalho apresenta um modelo para estudo da difusão da tecnologia móvel celular. Baseado na teoria de difusão de produtos de Frank Bass (1969), e na metodologia de Dinâmica de Sistemas, o modelo foi aplicado ao caso brasileiro. Como resultado, foi possível reproduzir, com bom grau de aderência, as curvas acumuladas de celulares adotados no Brasil (pós-pagos, pré-pagos e total), bem como a curva de vendas anuais. Os resultados das projeções apontam para uma saturação da penetração de telefones celulares no Brasil por volta do ano de 2013, quando então o mercado deverá atingir um total aproximado de 150 milhões de aparelhos.

Modelagem, simulação, dinâmica de sistemas, telefonia celular.

\section{Study of the cellular phone diffusion in Brazil: a system dynamics approach}

\begin{abstract}
This work presents a model to study the diffusion of the mobile cell phone technology. Based on the Frank Bass' theory of Products Diffusion (1969), and on the System Dynamics methodology, the model was applied to the Brazilian case. As a result, it was possible to reproduce, with a good degree of adherence, the accumulated curves of cellular adopted in Brazil (post-paid, pre-paid and total), as so the annual sales curve. The projected results indicates a saturation of cell phones diffusion in Brazil around the year 2013, when the market will supposed to reach a total of approximately 150 millions of devices.
\end{abstract}

\section{KEY WORDS}

Modeling, simulation, system dynamics, cell phone technology. 


\section{INTRODUÇÃO}

\subsection{Difusão de novos produtos - 0 modelo de Frank Bass}

Em geral, o lançamento de novos produtos afeta a vida de todos os indivíduos, comunidades e países. Todavia, para que um novo produto ou tecnologia se torne economicamente viável, é necessário um profundo conhecimento não apenas dos processos de desenvolvimento técnico, mas também de seus estágios de penetração e difusão nos mercados consumidores.

O termo difusão pode ser definido como o crescimento do uso ou consumo de um bem ou serviço dentro de um determinado grupo social (BROWN, 1981; STONEMAN, 2002). Este assunto tem sido abordado desde a década de 1960 por diversos autores (HAUSER et al., 2006). Um deles, Frank Bass (1969), propôs um modelo matemático que visava descrever a evolução, ao longo do tempo, do número de consumidores que adotavam um determinado produto desde o seu lançamento. O modelo de Bass é um dos modelos matemáticos mais utilizados no estudo da difusão de novos produtos. Sua construção e seu comportamento racional são consistentes com diversos estudos no campo da ciência social, e sua eficácia tem sido extensivamente comprovada ao longo dos anos (WRIGHT; CHARIETT, 1995).

O modelo de Bass nasce de uma hipótese comportamental na qual se assume que, durante o processo de difusão do uso de um novo produto, dois tipos de consumidores irão determinar a forma como a demanda irá crescer. O primeiro tipo corresponde àqueles indivíduos que decidem adotar uma inovação de forma independente, ou seja, não recebem influência direta de outros consumidores, todavia, podem ser motivados por outros meios, por exemplo, por comunicação de massa. Esses indivíduos são denominados "inovadores". $\mathrm{O}$ segundo tipo corresponde aos consumidores potenciais que são influenciados pela pressão social do meio e que são susceptíveis à influência de outros consumidores que já adquiriram o produto. Estes consumidores são denominados "imitadores".

Essa hipótese comportamental pode ser transcrita diretamente em um modelo matemático. Considere $S(t)$ como sendo o total acumulado no instante $t$ de consumidores que já adquiriram um determinado produto (primeira aquisição), em um mercado de $m$ consumidores potenciais. No modelo de Bass assume-se de forma simplificada que a "pressão de adoção" - $P(t)$, que corresponde à probabilidade de compra no instante $t$, por um consumidor aleatório, é uma função linear da proporção de usuários que já adotaram o produto, ou seja:

$$
P(t)=p+q \cdot \frac{S(t)}{m} .
$$

A Equação (1) corresponde à premissa fundamental do modelo de Bass. Se verdadeira, então o parâmetro $p$ corresponderá à tendência autônoma (intrínseca) do indivíduo em adotar o novo produto, geralmente estimulada pelo processo de comunicação (HORSKY; SIMON, 1983; KALISH; LILIEN, 1986; KALISH, 1985). Esse parâmetro é comumente chamado na literatura de "coeficiente de inovação". Já o parâmetro $q$ é um parâmetro que está ligado a uma tendência de adoção motivada pelo chamado "contágio social".

Observe que o segundo termo da Equação (1) crescerá na medida em que aumentar a proporção de indivíduos no mercado que adotam o produto, $(\mathrm{S}(t) / \mathrm{m})$, e este crescimento será proporcional ao parâmetro $q$. O parâmetro $q$ corresponde então a uma força de contágio, e é por isso que é chamado na literatura de "coeficiente de imitação".

Uma vez que $S(t)$ é o total acumulado no instante $t$ de consumidores que já adquiriram um determinado produto, $d S(t) / d t$ será a taxa de acréscimo de novos consumidores a cada instante de tempo. Logo, a proporção de indivíduos no instante $t$ que adotam o produto, em relação àqueles que ainda não adotaram no mercado, poderá ser dada por:

$$
h(t)=\left(\frac{1}{m-S(t)}\right) \cdot \frac{d}{d t} S(t) .
$$

Onde $m$ corresponde ao número total de consumidores potenciais no mercado.

Combinando a Equação (2) com a Equação (1), e definido $U(t)=(m-S(t))$ como sendo o número de consumidores potenciais que ainda restam adquirir o produto, obtém-se o modelo de Bass na sua forma diferencial:

$$
\frac{d}{d t} S(t)=p \cdot U(t)+q \cdot U(t) \cdot \frac{S(t)}{m} .
$$

A curva $S(t)$ obtida pela solução da Equação (3) apresenta um padrão de crescimento logístico com saturação em $S(t)=m$ (Figura 1). Em razão disto, $m$ também é chamado de "capacidade de carga" do mercado.

\subsection{Telefonia celular no Brasil}

A difusão do uso da telefonia celular vem crescendo rapidamente no mundo inteiro. No Brasil, dados da Agência Nacional de Telecomunicações (ANATEL) mostraram que em 2006 o número acumulado de telefones celulares atingiu a cifra aproximada de 100 milhões de aparelhos (Tabela 1). 
Além disso, a velocidade de difusão chegou a atingir patamares de crescimento de mais de $50 \%$ ao ano.

Rápidas taxas de crescimento como as observadas nos dados da Tabela 1 são bastante freqüentes em fenômenos econômicos que parecem obedecer a um processo do tipo “contágio" (VAN DEN BULTE; STREMERSCH, 2004). Ou seja, o número de usuários cresce proporcionalmente ao número de usuários já existentes, levando a um rápido aumento exponencial no início, quando do lançamento do produto, e a um posterior declínio da taxa de crescimento devido a uma saturação do mercado. Essas são características típicas das soluções do modelo de difusão de Bass (vide Figura 1).

Tabela 1: Número de telefones celulares no Brasil.

\begin{tabular}{cccc}
\hline \multicolumn{4}{c}{ Número de telefones celulares no Brasil } \\
\hline Ano & Pré-Pagos & Pós-Pagos & Total \\
\hline 1990 & - & 667 & 667 \\
1991 & - & 6.700 & 6.700 \\
1992 & - & 31.726 & 31.726 \\
1993 & - & 191.402 & 191.402 \\
1994 & - & 755.224 & 755.224 \\
1995 & - & 1.416 .500 & 1.416 .500 \\
1996 & - & 2.744 .549 & 2.744 .549 \\
1997 & - & 4.550 .175 & 4.550 .175 \\
1998 & - & 7.368 .218 & 7.368 .218 \\
1999 & $5.733 .743^{*}$ & $9.298 .954^{*}$ & 15.032 .698 \\
2000 & $13.606 .751^{*}$ & $9.581 .419^{*}$ & 23.188 .171 \\
2001 & $19.468 .405^{*}$ & $9.277 .363^{*}$ & 28.745 .769 \\
2002 & 25.002 .071 & 9.878 .893 & 34.880 .967 \\
2003 & 35.357 .004 & 11.016 .262 & 46.373 .266 \\
2004 & 52.793 .488 & 12.812 .089 & 65.605 .577 \\
2005 & 69.670 .050 & 16.540 .286 & 86.210 .336 \\
2006 & 80.555 .682 & 19.362 .939 & 99.918 .621 \\
\hline
\end{tabular}

* Dados estimados (fonte: ANATEL. Rel. de Acomp. e Avaliação 2002 Figura 11).

Fonte: Anatel

O objetivo deste trabalho é o de descrever, com o uso de um modelo matemático, o processo de difusão da telefonia celular no Brasil. Para alcançar este objetivo, utilizou-se a equação de Bass (Equação (3)), exposta na seção anterior, e a metodologia de Dinâmica de sistemas, que será exposta na próxima seção.

Como é um setor onde os processos de evolução tecnoló- gica são contínuos, levando a grandes investimentos técnicos e de infraestrutura, as operadoras deste tipo de serviço necessitam frequentemente efetuar estudos sobre a evolução da demanda para formular estratégias de criação de oferta. Dessa forma, o modelo apresentado neste trabalho poderá auxiliar no dimensionamento de investimentos futuros a serem realizados por essas empresas.

\section{A METODOLOGIA DE DINÂMICA DE SISTEMAS}

\subsection{Pensamento sistêmico e modelos mentais}

É inegável a importância do pensamento sistêmico no processo de planejamento e administração (GEORGANTZAS; ACAR, 1995). Para que um determinado projeto possa ser avaliado, seus resultados devem ser antecipados e considerados de acordo com as possíveis mudanças no ambiente. Para compreender essas mudanças, na maioria das vezes tenta-se analisar o problema desmontando-o em partes para depois examinar essas partes e tentar compreender o todo. Todavia, a empresa e seu ambiente constituem-se em parte de um sistema. Um sistema é um conjunto de duas ou mais entidades ou elementos, cada qual tendo um papel de atuação sobre o todo do sistema. O efeito de cada entidade depende dos efeitos causados por todas as demais entidades. Os sistemas apresentam propriedades que nenhuma de suas partes isoladamente poderia apresentar. Como consequência, um sistema não pode ser compreendido simplesmente dissecando-o, o que significa analisá-lo por partes. Para compreender completamente um sistema, deve-se identificar o todo, o contexto do qual o sistema faz parte, explicar o comportamento desse todo, e então explicar o comportamento do sistema em questão. Nos processos de planejamento nas empresas, a análise dos sistemas subjacentes deveria se tornar tão importante quanto a análise do comportamento das variáveis críticas do negócio, o que infelizmente não ocorre hoje, pelo menos em muitos casos.

A abordagem sistêmica do processo de planejamento tem em geral como ponto de partida a construção dos modelos mentais que descrevem esses sistemas (SENGE, 1994). Tais modelos são representações das estruturas subjacentes aos sistemas organizacionais, econômicos ou sociais que estão sendo analisados. Procura-se com eles examinar a interrelação das forças estruturais em um contexto mais amplo que permita entendê-las como parte de um processo comum (PIDD, 1998).

Em casos práticos, a construção e o trabalho com modelos mentais pressupõem o uso de alguma ferramenta de Mapeamento Cognitivo (PIDD, 1998). O Mapeamento Cognitivo (EDEN, 1988) faz parte de um conjunto de metodologias 
que dá corpo ao que se convencionou denominar Abordagem Soft da Pesquisa Operacional, voltada muito mais para a estruturação de problemas complexos, auxiliando no discernimento de soluções, do que para sua resolução propriamente dita. Essas técnicas de mapeamento procuram representar a percepção dos indivíduos envolvidos em uma situação problemática e fundamentam-se principalmente na Teoria dos Constructos Pessoais da área de psicologia (KELLY, 1955; PIDD, 1998).

Uma importante ferramenta de Mapeamento Cognitivo são os chamados Diagramas Causais da Dinâmica de Sistemas (STERMAN, 2000). Os Diagramas Causais são diagramas que relacionam duas ou mais entidades de um sistema especificando que variações associadas a uma entidade irão gerar variações associadas a outra entidade. Ou seja, uma relação é dita causal se a ocorrência de um primeiro evento for condição necessária para a ocorrência de um segundo evento. Os diagramas causais são diagramas em que as entidades do sistema aparecem interligadas por setas representando as relações de causalidade. $\mathrm{O}$ sentido das setas indica o sentido da relação de causalidade. Além disso, cada seta pode possuir um sinal (+ ou -) indicando se as mudanças nas variáveis de causa e efeito têm o mesmo sentido $(+)$ ou sentido contrário (-).

Como exemplo, considere o seguinte caso (adaptado de GEORGANTZAS; ACAR, 1995, p. 80): Uma empresa de prestação de serviços utiliza recursos de tecnologia de informação para disponibilizar para seus clientes, acesso estruturado e privilegiado a várias bases de dados de pesquisa na área de negócios. A capacidade de atendimento da empresa depende dos recursos disponíveis (pessoas, equipamentos, linhas de comunicação, etc.) que derivam de investimentos externos feitos. Entende-se que esta capaci- dade tem impacto direto na demanda gerada, sendo que a demanda, aqui, corresponde às requisições de novos clientes e dos parceiros de conteúdo. Na medida em que as vendas da empresa crescem, isto impacta positivamente na imagem da empresa (dentro da região onde ela atua), gerando um impacto positivo na demanda. Por fim, o aumento da demanda irá gerar vendas, que por sua vez irão gerar resultados que eventualmente serão convertidos em recursos. O diagrama causal resultante desse caso pode ser visto na Figura 2. Esse diagrama busca representar uma visão situacional das relações existentes e sua construção deve resultar em um diagrama claramente interpretado por todos os tomadores de decisão envolvidos em sua construção.

\subsection{Modelos formais e simulação}

Apesar de sua importância para a representação dos modelos mentais dos participantes de um processo de planeja-

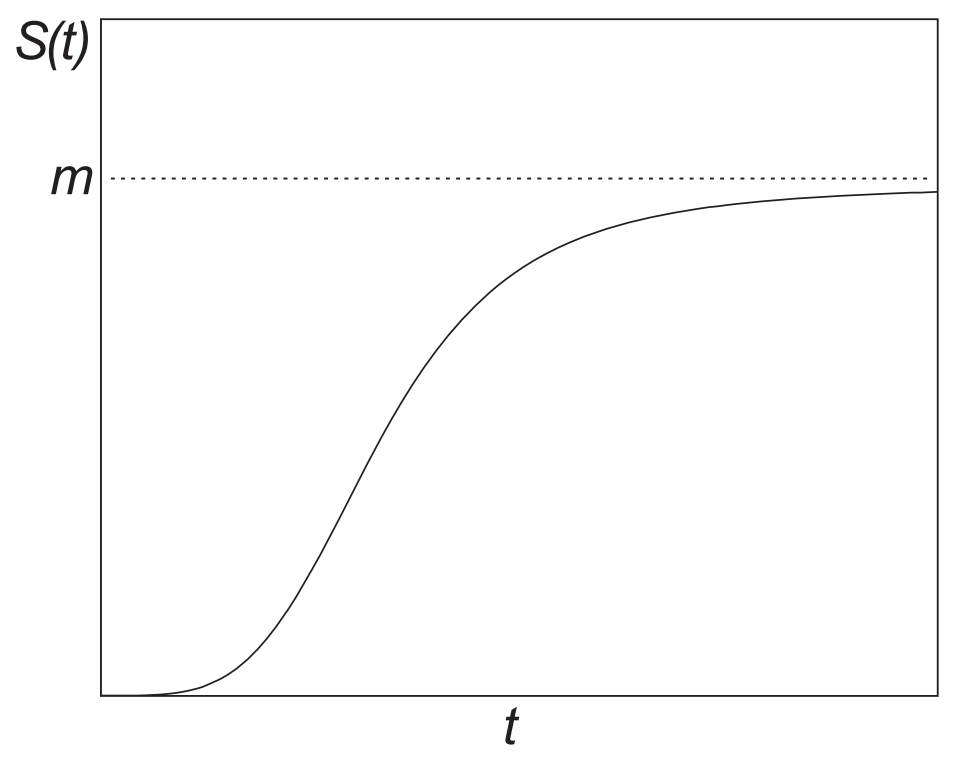

Figura 1: Representação genérica de uma solução da Equação (3). 
mento, os diagramas causais falham em responder a questões tais como: Que variável é mais decisiva e impactante para o funcionamento do sistema? Qual variável possui papel dominante para o sucesso de uma determinada estratégia? Quais os impactos das variações externas sobre o ambiente interno da empresa?
A partir dos modelos criados, podem-se definir vários cenários e testar seus impactos sobre o comportamento do sistema (FAHEY; RANDALL, 1998).

A construção de um modelo formal, que seja voltado para a simulação, pressupõe o conhecimento explícito das relações quantitativas entre as variáveis do modelo causal. Isto tem sido um empecilho para o uso mais efetivo de tais modelos nos processos de planejamento. A necessidade de especialistas em modelagem quantitativa $\mathrm{e}$ a dificuldade no levantamento preciso de todas as relações quantitativas consomem muito tempo e dinheiro. A Metodologia de Dinâmica de Sistemas tem ajudado a atacar

Para dar respostas para essas questões, deve-se desenvolver um processo de modelagem formal. Modelos formais são basicamente modelos baseados em conceitos matemáticos e computacionais (PIDD, 1998). A abordagem formal abre as portas para o desenvolvimento de capacidades de simulação, e assim pode representar um bom ponto de partida para a modelagem estratégica através da simulação de cenários.

O uso prático e funcional das análises causais pressupõe o uso de modelagem computacional e simulação (FORRESTER, 1969; ROBERTS et al., 1983; FAHEY; RANDALL, 1998; STERMAN, 2000). Isso permitirá a avaliação dos efeitos das alterações nas variáveis externas e internas que afetam a situação de uma empresa. Torna-se possível a experimentação de alternativas e seu acompanhamento por meio da visualização do comportamento dessas variáveis. parte desses problemas.

A Dinâmica de Sistemas é uma metodologia ligada ao pensamento sistêmico, que busca estudar as estruturas dos sistemas organizacionais e sociais por meio da representação das relações causais entre os seus elementos e por meio do estudo de sua evolução ao longo do tempo. O principal objetivo é explicar o comportamento de um determinado sistema partindo das interações entre as diversas partes que o compõem, e utilizando padrões de comportamento existentes (STERMAN, 2000; PIDD, 1998; FORRESTER, 1961).

Por meio da utilização de ferramentas e ambientes computacionais de simulação, permite testar diferentes políticas e soluções para a operação de um sistema, avaliando o im-

\subsection{Dinâmica de Sistemas: Diagramas de estoque e fluxo}

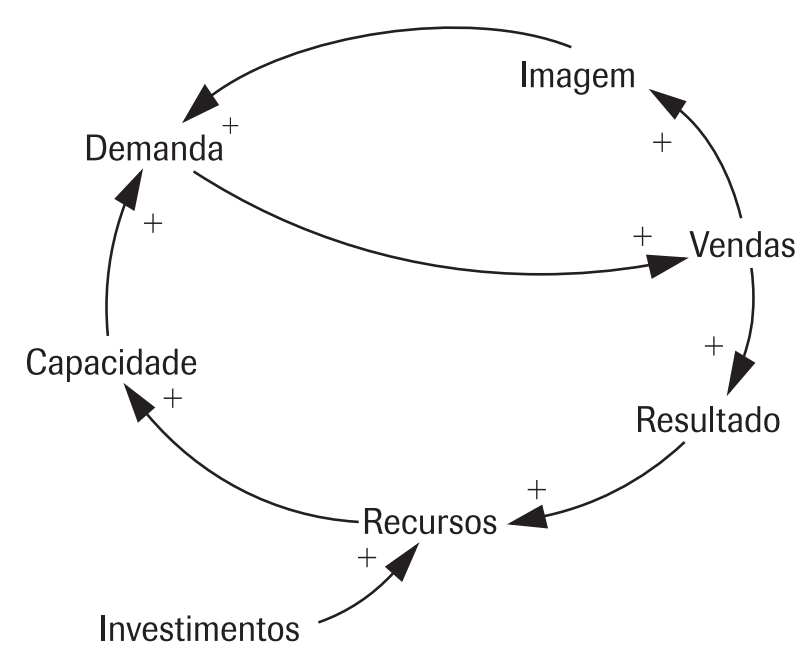

Figura 2: Exemplo de modelo mental (Diagrama Causal). 
pacto de suas decisões e proporcionando um conjunto de instrumentos para compreensão e comunicação sobre os modelos construídos (PIDD, 1998). A Dinâmica de Sistemas assume que a análise de uma situação pode ser empreendida de um ponto de vista externo ao sistema, e que a estrutura e os processos dinâmicos podem ser recriados em diagramas com simbologia específica e modelos matemáticos apropriados.

Em Dinâmica de Sistemas a idéia fundamental é que o comportamento dinâmico de um sistema obedece a um princípio chamado de "princípio da acumulação". Este princípio postula que a resposta dinâmica de um sistema decorre da transição dos valores de seus recursos acumulados em estoques e que essas transições são controladas por fluxos de entrada e saída nos estoques. Ou seja, o comportamento dinâmico surge quando algo flui por algum meio. Este princípio leva a uma forma particular de representação causal conhecida por Diagramas de Estoque e Fluxo (STERMAN, 2000).

Nestes diagramas os estoques, ou níveis, são representados graficamente por retângulos. Eles representam caixas onde se acumula algum recurso do sistema. Os estoques são variáveis cujo valor, ou estado, depende dos fluxos. Os fluxos são representados por setas de traço duplo que são cortadas por um triângulo. Os fluxos representam o transporte dos recursos dentro do sistema. Os fluxos são vazões que são controladas por equações e por isto são representados por um ícone parecido com um cano com uma torneira. A Figura 3 mostra a representação básica de um estoque controlado por um fluxo em Dinâmica de Sistemas (a nuvem em uma das pontas da seta representa uma fonte infinita alimentando o fluxo).

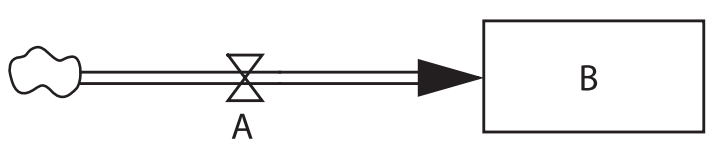

Figura 3: Estoque $(B)$ controlado por um Fluxo $(A)$. Fonte: o autor.

Na Figura 3 o valor do estoque $B$ é controlado pelo fluxo $A$. Este controle pode ser expresso através de uma equação diferencial que descreve as transições dos valores do estoque $B$ entre os instantes $t$ e $t+d t$. Estas transições são descritas pela equação (4):

$$
B(t+d t)=B(t)+A(t) \cdot d t \Rightarrow A(t)=\frac{d B}{d t} .
$$

$\mathrm{Na}$ modelagem com Diagramas de Estoque e Fluxo, tanto variáveis físicas como de informação podem fluir pelos fluxos, acumulando-se em estoques, e este acúmulo é decorrente da diferença entre os fluxos de entrada e os fluxos de saída. Logo, os recursos ao longo do tempo não podem mudar instantaneamente de valor.

\section{A resposta dinâmica de um sistema decorre da transição dos valores de seus recursos acumulados em estoques.}

Exemplos de estoque podem ser o volume de produção de uma empresa, o número de peças nos estoques, o total de dinheiro no caixa, o conhecimento dos funcionários, o número de consumidores no mercado, etc. Exemplos de fluxos podem ser a taxa de produção da empresa, as taxas de vendas, o volume mensal de pagamentos, etc. No modelo que iremos apresentar neste trabalho, os estoques representarão estoques de não consumidores e de consumidores de telefones celulares no mercado brasileiro e os fluxos serão fluxos de vendas de celulares e de conversão de usuários, conforme explicaremos mais à frente.

Os valores futuros dos estoques devem ser o foco das decisões dos gestores. Os fluxos representam as políticas do sistema, e podem ser descritos na forma de equações algébricas. Estas equações podem envolver variáveis auxiliares e, ou, o valor atual dos estoques (MORECROFT; STERMAN, 2000). É importante notar que os fluxos atuais de um sistema são modificados por ações presentes que decorrem de decisões presentes, enquanto que os estoques atuais dependem do acúmulo de todas as decisões passadas (FORRESTER, 1969).

As principais vantagens advindas do uso da metodologia de Dinâmica de Sistemas provêm de sua capacidade de: a) Investigar as relações entre macro e microestruturas e seus efeitos sobre o comportamento do sistema; b) Modelar e resolver problemas reais, incorporando fatores biológicos, físicos e econômicos; c) Melhorar o desempenho de um sistema via adição de "insights" ou aprendizagem, aliado ao melhor uso dos recursos; d) Estudar os fluxos de material, informação e dinheiro dentro de estruturas econômicas; e e) Não ter um limite dos problemas que possa abranger, podendo captar situações de equilíbrio, desequilíbrio e até mesmo comportamentos caóticos.

Frente a estas habilidades, a metodologia de Dinâmica de Sistemas vem sendo aplicada nas mais diversas áreas do conhecimento. Neste trabalho será feito uso dela como 
instrumento para investigação, simulação, previsão e aprendizado na área de difusão de tecnologias.

\section{DESENVOLVIMENTO DO MODELO}

Considere o caso em que o número de consumidores no modelo de Bass (Equação (3)) que adotaram e os que eventualmente ainda podem adotar o uso de um determinado produto ou tecnologia é representado por estoques, de acordo com os modelos de Dinâmica de Sistemas.

Na Figura 4 tem-se uma representação em Diagramas de Estoque e Fluxo do modelo de Bass. As relações causais entre o fluxo $D S d t$ e os parâmetros $p, q$ e $m$ são chamadas de relações de transmissão de informação. As relações de transmissão de informação, representadas por setas de traço simples, ligam elementos do sistema explicitando relações entre os mesmos, mas, diferente dos fluxos, não retiram ou colocam recursos nos estoques.

O processo de modelagem em Dinâmica de Sistemas pressupõe a existência de ambientes computacionais próprios para a construção dos modelos e execução das simulações. Várias são hoje as plataformas que permitem a implementação de modelos com o uso dessa metodologia. A mais conhecida e utilizada destas plataformas é o software Vensim, da Ventana Systems (VENSIM, 2007). Isso se deve principalmente ao fato de sua versão PLE (disponibilizada através da Internet) ser gratuita para fins acadêmicos e educacionais. Neste trabalho, os modelos apresentados foram desenvolvidos com o uso do software Vensim.

No caso da Figura 4, as equações no Vensim que descrevem quantitativamente as relações entre as variáveis e parâmetros são registradas computacionalmente da seguinte forma:

$$
\begin{aligned}
& \text { DSdt }=\mathrm{p}^{*} \mathrm{U}+\mathrm{q} * \mathrm{U} *(\mathrm{~S} / \mathrm{m}) \\
& \mathrm{U}=\text { INTEG }(-\mathrm{DSd} \mathrm{t}) \\
& \mathrm{F}=\operatorname{INTEG}(\mathrm{DSdt})
\end{aligned}
$$

A construção de um modelo computacional que resolva no tempo o conjunto de Equações (5) nem sempre é fácil. Felizmente as ferramentas de simulação de Dinâmica de Sistemas já são desenhadas para implementar de maneira natural estruturas como as da Figura 4. Estão preparadas para integrar numericamente os fluxos para a obtenção dos valores dos estoques. Sendo assim, cabe ao modelista especificar as funções que representam cada variável e deixar que o sistema se encarregue da solução numérica (VENSIM, 2007).

O modelo desenvolvido neste trabalho pode ser visto na Figura 5.

Na Figura 5 o estoque $U$ corresponde ao número de consumidores, em um mercado potencial de tamanho $m$, que ainda não adquiriram pela primeira vez um telefone celular. Este estoque é feito inicialmente igual a $m$. O estoque de consumidores $U$ alimenta dois outros estoques: o estoque de adotantes de pós-pagos (Pospagos) e o estoque de adotantes de pré-pagos (Prepagos). Os fluxos VendasPre e VendasPos são definidos a partir do modelo de difusão de Bass. No caso do fluxo VendasPre, este só é acionado após o ano de lançamento dos pré-pagos, ou seja, em $T_{1}=1999$, e é dado por:

VendasPre $(t)= \begin{cases}0 & \text { se } T_{0} \leq t<T_{1} \\ p_{2} \cdot U(t)+q_{2} \cdot U(t) \cdot \frac{N(t)}{m} & \text { se } t \geq T_{1}\end{cases}$

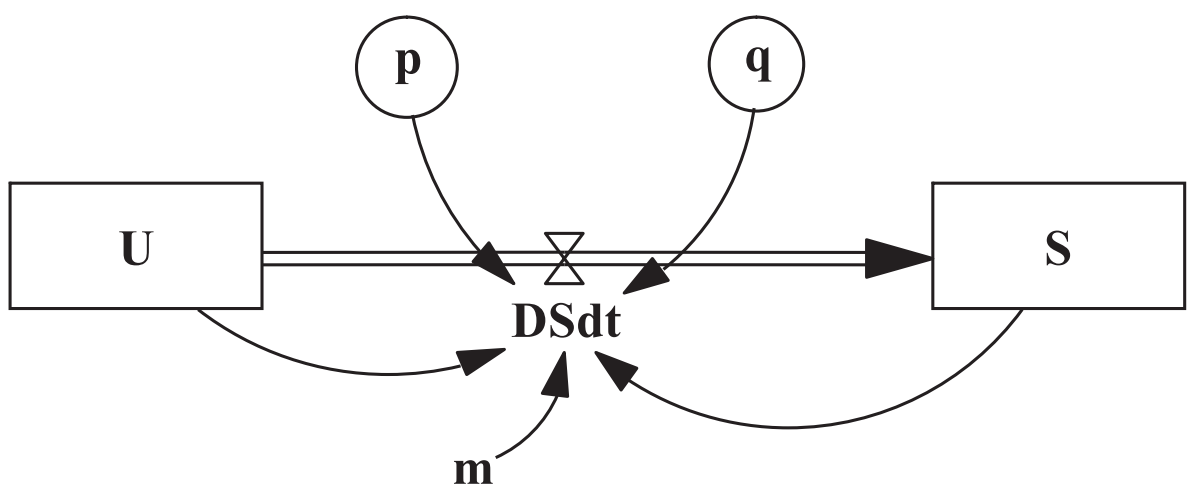

Figura 4: Representação do modelo de Bass (Equação (3)) em Diagramas de Estoque e Fluxo. 
Onde $T_{0}=1990$ é o ano de início de registro de vendas de celulares e $N(t)=(\operatorname{Pospagos}(t)+\operatorname{Prepagos}(t))$ é o número total de usuários que já adquiriram um telefone celular.

O fluxo VendasPos é dado pela Equação (7). Ao observar esta equação, vê-se que no intervalo de tempo entre $T_{0}=$ 1990 e $T_{1}=1999$, o fluxo VendasPos é definido de acordo com o modelo clássico de Bass, todavia, após o lançamento dos pré-pagos, supõe-se haver um período de transição no processo de difusão dos telefones pós-pagos, ocasionado pela entrada da tecnologia dos pré-pagos. Esse período de transição tem sua duração dada por $\left(T_{2}-T_{1}\right)$. Uma vez que se conhece o valor de $T_{1}=1999, T_{2}$ torna-se um parâmetro de ajuste numérico do modelo.

$\operatorname{VendasPos}(t)= \begin{cases}p_{0} \cdot U(t)+q_{0} \cdot U(t) \cdot \frac{N(t)}{m} & \text { se } T_{0} \leq t<T_{1} \\ U(t) \cdot w & \text { se } T_{1} \leq t \leq T_{2} \\ p_{1} \cdot U(t)+q_{1} \cdot U(t) \cdot \frac{N(t)}{m} & \text { se } t>T_{2}\end{cases}$

Durante o período de transição assume-se, por simplicidade, que a venda de pós-pagos é proporcional ao número ainda existente de consumidores potenciais $U(t)$. O parâmetro de proporcionalidade, $w$, também será ajustado numericamente utilizando os dados da ANATEL (Tabela 1). Após $T_{2}$, com a consolidação do uso da tecnologia dos pré-pagos, é assumido que a difusão no mercado de pós-pagos volta a se comportar de acordo com o modelo de Bass, porém com novos coeficientes.

Finalmente, supõe-se que há um terceiro fluxo no modelo (Trocas). Este fluxo alimenta o estoque de usuários de prépagos e corresponde aos usuários que realizaram uma troca de tecnologia, migrando de pós-pago para pré-pago. O fluxo Trocas é assumido como sendo proporcional ao número de usuários de pós-pagos, todavia, ponderado por uma distribuição gaussiana centrada em um instante de tempo $T_{3}>T_{1}$ e com dispersão $d$. Isso significa que as trocas de pós-pagos para pré-pagos começam a ocorrer de forma gradual após o lançamento dos pré-pagos, e atingem um máximo em $\mathrm{t}=T_{3}$, voltando a cair depois com dispersão $d$.

$$
\operatorname{Trocas}(t)=\left\{\begin{array}{ll}
0 & \text { se } t<T_{1} \\
\operatorname{Pospagos}(t) \cdot k \cdot e^{-\frac{1}{d^{2}}\left(t-T_{3}\right)^{2}} & \text { se } t \geq T_{1}
\end{array} .\right.
$$

Uma vez definidas as equações do modelo, restam 12 parâmetros que devem ser ajustados em relação aos dados da ANATEL (Tabela 1). São eles: $m, p_{0}, p_{1}, p_{2}, q_{0}, q_{1}, q_{2}, k$, $w, d, T_{2}$ e $T_{3}$. Para realizar o ajuste do modelo, foram feitas 200.000 simulações utilizando-se o método de otimização de Powell (2000). Os valores que minimizaram o erro quadrático médio, em relação aos dados da ANATEL estão apresentados na Tabela 2.

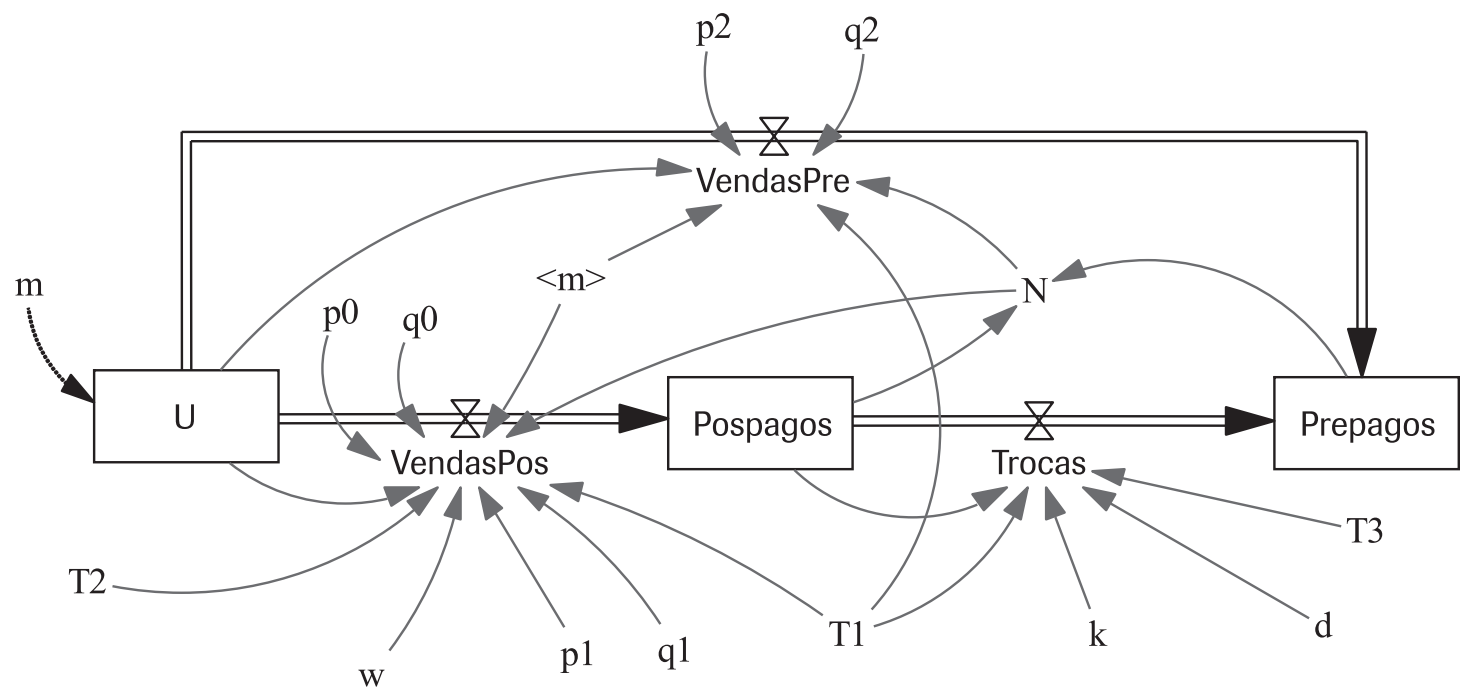

Figura 5: Modelo desenvolvido para estudar a difusão de telefones celulares no Brasil. 
Tabela 2: Parâmetros obtidos do ajuste do modelo em relação aos dados da ANATEL.

\begin{tabular}{cc}
\hline \multicolumn{2}{c}{ Parâmetros ajustados do modelo } \\
\hline$m$ & $157,39 \mathrm{E} 6$ \\
$p 1$ & 0,000203 \\
$p 2$ & 0,000001 \\
$q 0$ & 0,005639 \\
$q 1$ & 0,560398 \\
$q 2$ & 0,118191 \\
$w$ & 0,336407 \\
$k$ & 0,005557 \\
$T 2$ & 0,666716 \\
$T 3$ & 2003,018 \\
$d$ & 2003,967 \\
\hline
\end{tabular}

\section{RESULTADOS}

Todos os resultados apresentados nesta seção foram obtidos a partir da simulação feita utilizando os valores dos parâmetros vistos na Tabela 2. O método de integração numérica utilizado foi o Runge-Kutta, selecionado dentro do Vensim como método numérico para a integração das equações do modelo. O modelo simulado com esses parâmetros será daqui por diante chamado de "modelo ajustado.'

A Figura 6 mostra uma comparação entre o resultado do modelo ajustado e os dados reais do total acumulado de celulares no Brasil. O coeficiente de explicação obtido foi $\mathrm{R}^{2}=0,998$. Apesar de o resultado deste ajuste individual poder ser considerado bom, ele deve ser verificado de forma conjunta com outros resultados comparativos das saídas do modelo ajustado, pois o modelo apresenta um número grande de graus de liberdade de ajuste.

Na Figura 7 e na Figura 8, respectivamente, são apresentados os dados da evolução do número de celulares prépagos e pós-pagos no Brasil. Esses dados são comparados à saída do modelo ajustado. Os coeficientes de explicação obtidos foram $\mathrm{R}^{2}=0,994$ para os pós-pagos e $\mathrm{R}^{2}=0,997$ para os pré-pagos.

Por fim, foi feita a comparação do fluxo de vendas anuais de novos telefones celulares no Brasil com a respectiva saída do modelo ajustado. O fluxo de vendas anuais, dado de extrema importância em situações gerenciais, pode ser obtido a partir da Tabela 1, calculando-se a variação, ano a ano, do total acumulado de telefones vendidos, o que corresponde à derivada em relação ao tempo das vendas acumuladas. A fim de atenuar flutuações geradas por este cálculo de derivada,

Total acumulado de celulares pós e pré-pagos (Brasil).

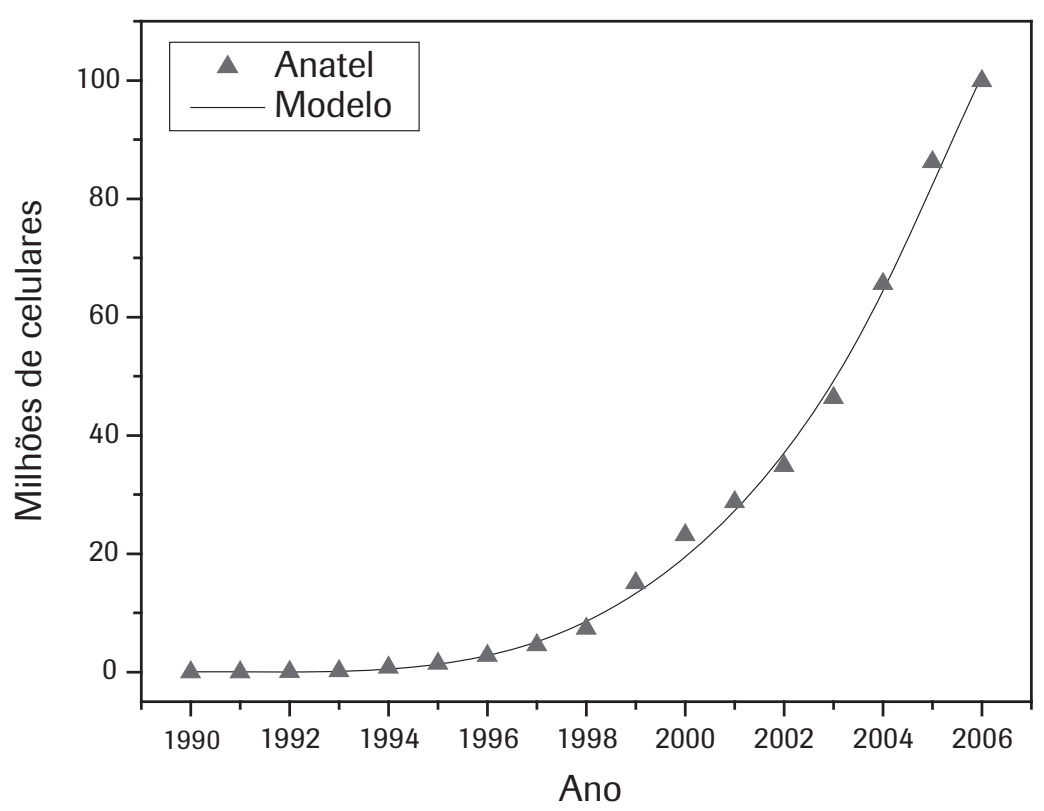

Figura 6: Total acumulado de celulares no Brasil. 
Total acumulado de celulares pré-pagos (Brasil) .

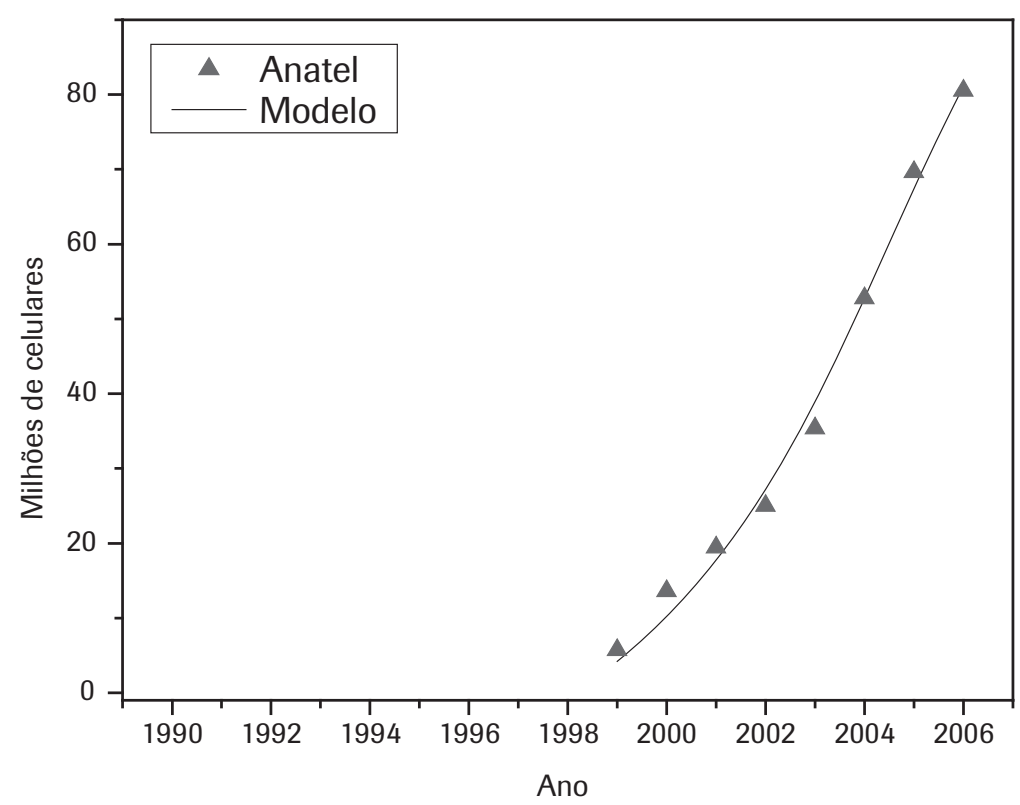

Figura 7: Total acumulado de celulares pré-pagos no Brasil.

Total acumulado de celulares pós-pagos (Brasil).

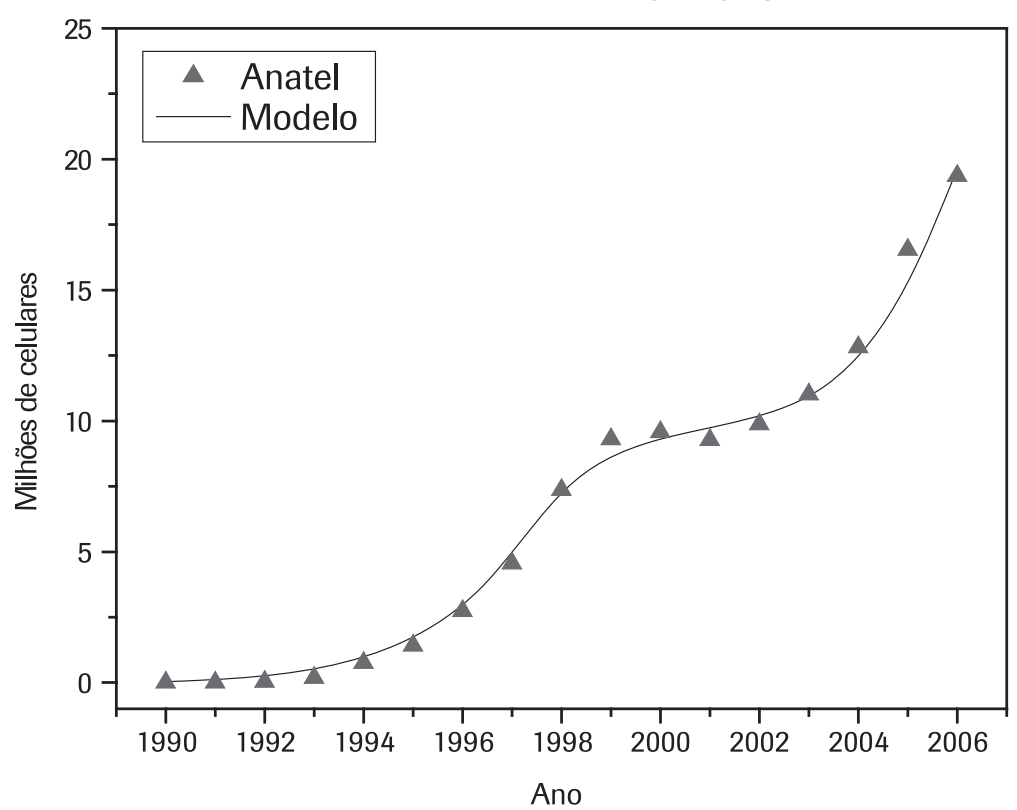

Figura 8: Total acumulado de celulares pós-pagos no Brasil. 
aplicou-se posteriormente uma média móvel de três meses ao resultado obtido.

Por fim, foi feita a comparação do fluxo de vendas anuais de novos telefones celulares no Brasil com a respec- da ANATEL e o resultado da simulação. O coeficiente de explicação obtido foi $\mathrm{R}^{2}=0,972$. A Figura 10 e a Figura 11 apresentam, respectivamente, os fluxos separados de vendas de pós e pré-pagos.

Percebe-se que na Figura 9 foi possível detectar uma inflexão na curva de vendas de novos telefones celulares. A inflexão ocorre durante o ano de 2005, ou seja, a partir desse ano, o modelo detectou o início de um eventual período de queda nas vendas. Essa observação será comentada com mais detalhes nas discussões dos resultados deste trabalho. tiva saída do modelo ajustado. O fluxo de vendas anuais, dado de extrema importância em situações gerenciais, pode ser obtido a partir da Tabela 1, calculando-se a variação, ano a ano, do total acumulado de telefones vendidos, o que corresponde à derivada em relação ao tempo das vendas acumuladas. A fim de atenuar flutuações geradas por este cálculo de derivada, aplicou-se posteriormente uma média móvel de três meses ao resultado obtido. No modelo desenvolvido, o fluxo de vendas anuais é obtido pela soma dos fluxos VendasPre e VendasPos.

O fluxo de vendas anuais é obtido pela soma dos fluxos VendasPre e VendasPos. A Figura 9 mostra o resultado comparativo entre o fluxo calculado a partir dos dados

\section{DISCUSSÃO DOS RESULTADOS}

Nesta seção, buscar-se-á discutir os resultados obtidos na seção anterior por meio da tentativa de responder a duas questões: Baseados na hipótese de validade do modelo ajustado, que cenários futuros podem ser traçados para a difusão da telefonia celular no Brasil, e como se pode compreender essa difusão com base nos parâmetros ajustados do modelo?

A resposta à primeira questão é mais bem entendida analisando-se a Figura 10 e a Figura 11. Na Figura 10 vê-se

Número de celulares vendidos a cada ano (Brasil).

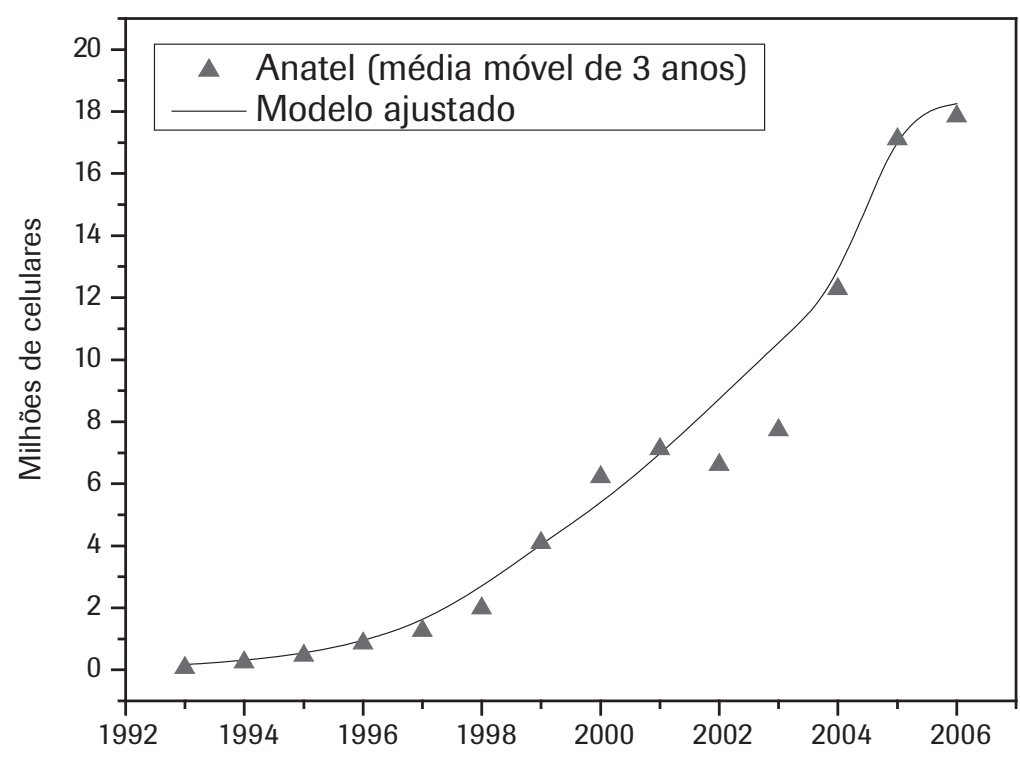

Figura 9: Média de novos celulares vendidos anualmente no Brasil. 
Número de celulares pós-pagos vendidos a cada ano (Brasil).

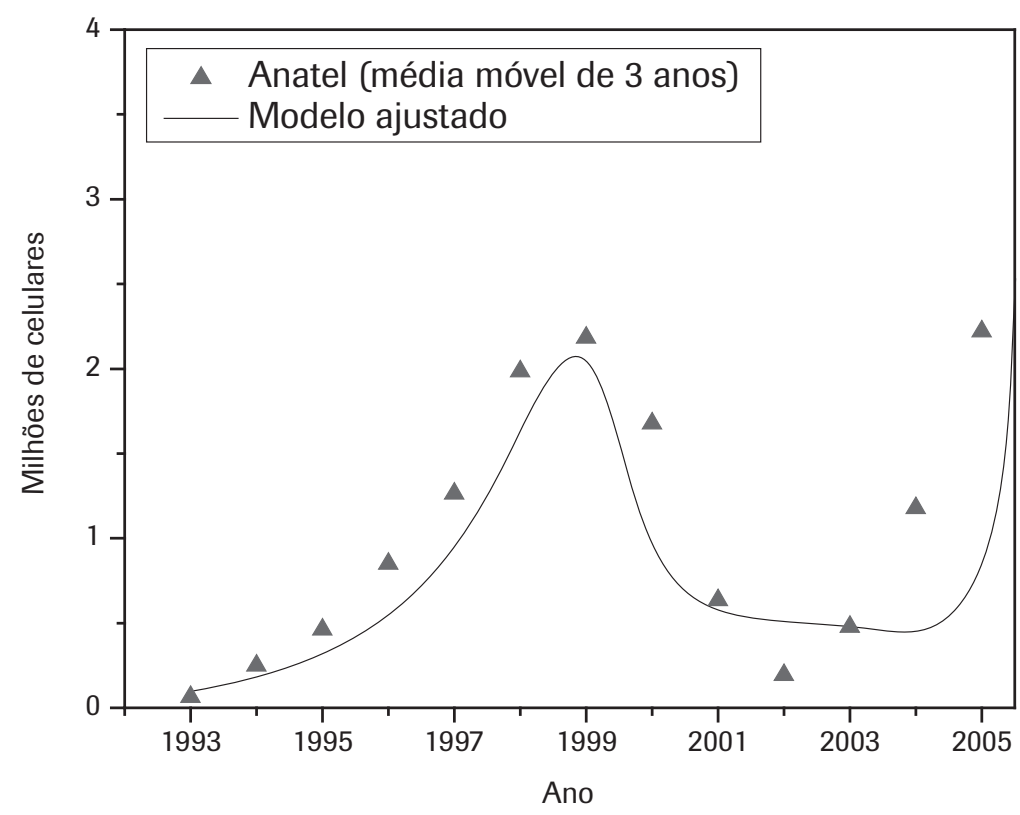

Figura 10: Média de pós-pagos vendidos anualmente no Brasil.

Número de celulares pré-pagos vendidos a cada ano (Brasil).

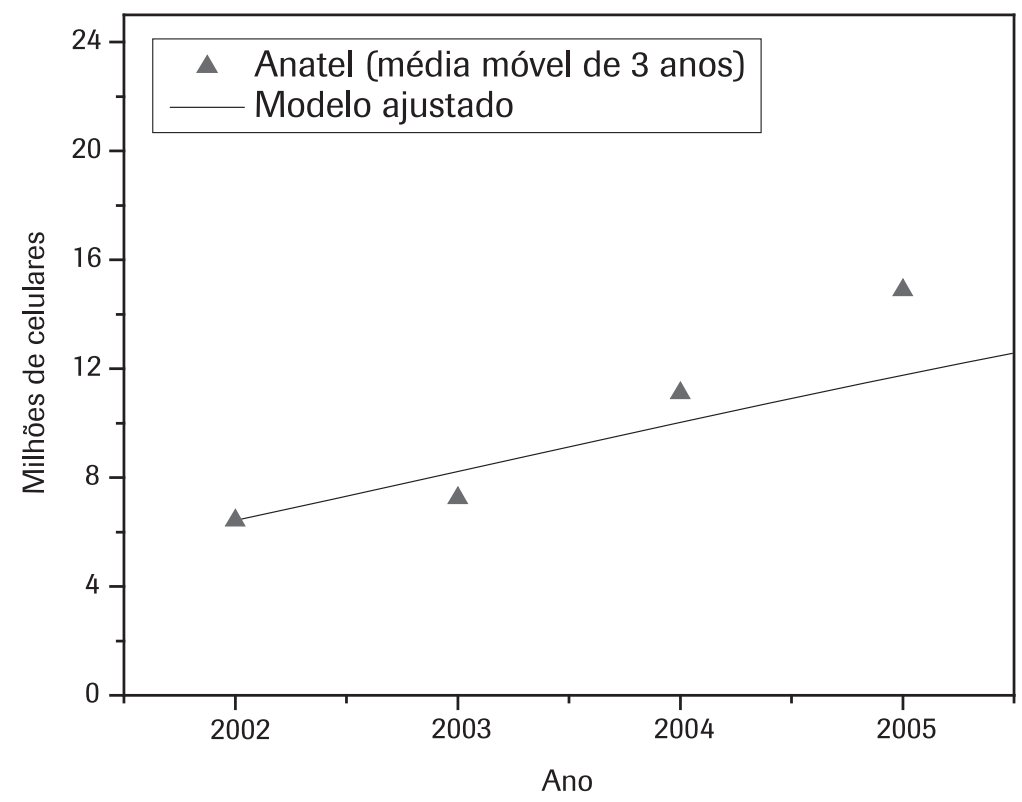

Figura 11: Média de pré-pagos vendidos anualmente no Brasil. 
uma projeção de cinco anos das vendas de celulares no Brasil, feita com o uso do modelo ajustado.

A projeção mostra que, a partir da segunda metade de 2005, as vendas de novos celulares começarão a cair. Sendo que, a partir de 2006, deverão cair a uma taxa aproximada de $20 \%$ ao ano durante os anos seguintes. Este é um resultado interessante, pois levanta a existência de um ponto de inflexão nas vendas, que marca o início de um período de quedas significativas nesse mercado.

O fato de presumidamente o país ter atingido seu ponto de inflexão de vendas de novos celulares não significa que não se tenha mais nenhum potencial de crescimento no setor. Ao observar a Figura 11, que apresenta uma previsão de cinco anos para o número total de celulares adquiridos, vêse que este número ainda deverá crescer, todavia, com uma velocidade menor, tendendo a atingir um patamar limite de aproximadamente 157 milhões de celulares. Por volta do ano de 2013 segundo os resultados da simulação.

Estes resultados podem ser mais bem entendidos ao analisarem-se os parâmetros obtidos pelo ajuste do modelo (Tabela 2). O que nos conduz à resposta da segunda questão colocada no início desta seção, sobre a interpretação dos parâmetros do modelo.

Na Tabela 2, o valor de $m \sim 157 \mathrm{M}$, correspondente à capacidade de carga ajustada do modelo, teoricamente representaria o limite de penetração desta tecnologia no mercado Brasileiro, e corresponderia em 2006 a uma taxa de penetração de aproximadamente $83 \%$ da população, tomando como base a estimativa de 2006 do IBGE que é de 188 milhões de habitantes. Essa é uma taxa de penetração bastante significativa, principalmente se for considerado o tamanho absoluto da população brasileira.

Ainda analisando os dados da Tabela 2, pode-se perceber que $p_{2} \gg p_{1}$ e que $q_{2} \gg q_{1}$. Ou seja, o coeficiente de inovação dos consumidores de pré-pagos é muito maior que o dos consumidores de pós-pagos, indicando que estes são muito mais susceptíveis à influência da comunicação. Da mesma forma, o coeficiente de imitação dos consumidores de pré-pagos é maior que o dos consumidores de pós-pagos, indicando que estes também são mais propensos a decidir a compra devido a "pressões" do meio social, ou seja, imitação. Todavia, essas constatações devem ser mais bem investigadas sob o ponto de vista das pesquisas mercadológicas e do comportamento de compra e consumo.

A característica presumida de que o consumidor de pré-pagos tem maior susceptibilidade à comunicação acaba fazendo com o que o mercado de pré-pagos apresente uma curva de penetração que atinge mais rapidamente o

Número de celulares vendidos a cada ano (Brasil).

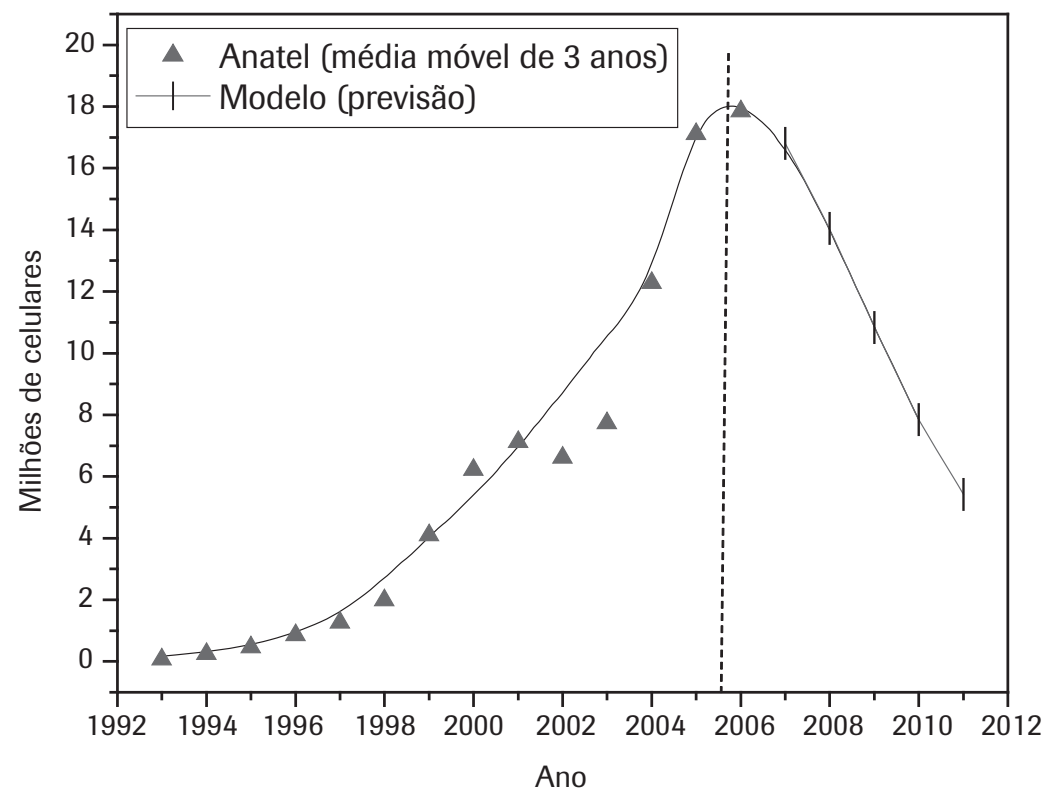

Figura 12: Média de novos celulares vendidos anualmente no Brasil. 
ponto de saturação. Isso ocorre por volta de dois anos antes da saturação do mercado de pós-pagos, de acordo com os resultados das simulações. Por fim, o fato de ter-se $q_{0}>q_{2}$ $>q_{1}$ é um forte indicativo de que, no início do processo de difusão dos telefones celulares no Brasil, a compra foi predominantemente orientada pela difusão "boca-a-boca", em um processo típico de contaminação e pressão social.

\section{CONCLUSÕES}

Este trabalho mostrou a capacidade que os modelos matemáticos de difusão têm para representar o processo de introdução de uma nova tecnologia, tal como a telefonia celular no Brasil. Em particular, mostrou que o uso da metodologia de Dinâmica de Sistemas como ferramenta de modelagem computacional permite o uso extensivo dos conceitos contidos no modelo de Bass em situações práticas de planejamento, voltadas tanto para o ambiente profissional quanto o de aprendizado.

Apesar das técnicas de modelagem e simulação apresentadas aqui terem sido desenvolvidas, em geral, como ferramentas para lidar com situações passíveis de ser matematicamente representadas, elas podem e devem ser exploradas também como processos para a captura, a estruturação e a compreensão de nossos modelos mentais e do nosso conhecimento. E este é um aspecto importante deste trabalho. Técnicas de modelagem, aliadas a formulações matemáticas objetivas, potencializam sua vocação como ferramentas de aprendizado e nos estimulam a testar os limites da nossa realidade.

Além disso, os resultados obtidos a partir da simulação nos permitem fazer análises mais profundas. Por exemplo, vimos que o coeficiente de imitação dos consumidores de pré-pagos obtido foi maior que o dos consumidores de pós-pagos. O coeficiente de imitação $q$ captura a efetividade da comunicação interpessoal como elemento propulsor da difusão. A literatura mostra que este coeficiente está basicamente relacionado com: (1) o nível de homogeneidade econômica e cultural da população e; (2) com a capacidade de persuasão dos consumidores que já adotaram o produto (TALUKDAR; SUDHIR; AINSLIE, 2002). Isso ocorre porque a interação pessoal e a comunicação boca a boca são muito mais facilitadas em mercados com populações homogêneas do que em mercados com populações mais heterogêneas (TAKADA; JAIN, 1991), e esse é o caso do mercado de linhas pré-pagas, basicamente composto por consumidores da base da pirâmide econômica, que em geral apresentam estratificação econômica bastante homogênea.

Total acumulado de celulares pós e pré-pagos (Brasil).

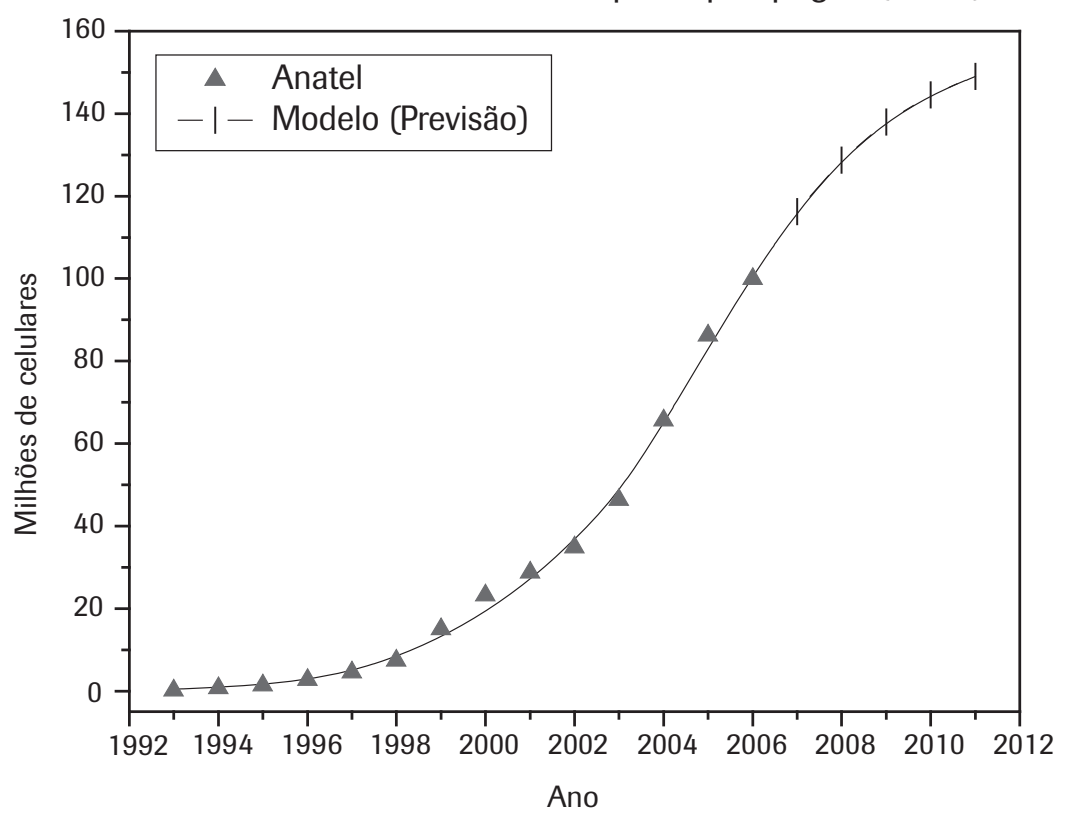

Figura 13: Total acumulado de celulares no Brasil. 
Outra situação que corrobora os resultados da simulação é o fato de que o coeficiente de imitação $q$ também é esperado ser maior em uma população em que seja maior a capacidade de persuasão das recomendações boca a boca dos adotantes existentes. Essa capacidade de persuasão das recomendações aumenta com uma maior familiaridade e satisfação dos atuais adotantes com o novo produto e quanto maior o nível de renda destes (TAKADA; JAIN, 1991). Encontramos no mercado brasileiro de telefonia móvel tal situação ao perceber que os primeiros influenciadores dos usuários de pré-pagos foram os usuários iniciais de pós-pagos, predominantemente, no início, das classes A e B. Consumidores com rendas mais altas são geralmente os primeiros a adotar um novo produto (ROGERS, 1995). A razão disto é que os consumidores mais ricos podem pagar mais por um novo produto comparativamente aos consumidores mais pobres, e também estão dispostos a assumir maiores riscos ao adotar um novo produto recém-lançado no mercado (DICKERSON; GENTRY, 1983). recido pelo produto que está sendo introduzido em relação ao produto, ou produtos, que no instante da introdução serviam às necessidades do consumidor (HORSKY, 1990). Ou seja, os consumidores podem ter se tornado mais propensos a mais rapidamente adotar um telefone celular, pelo fato de o acesso a linhas telefônicas fixas ser bastante deficiente. De maneira similar, o consumidor tende a trocar um telefone celular póspago por um pré-pago caso não perceba um benefício incremental gerado pela utilização do serviço pós-pago.

Muitas outras discussões e testes podem ser feitos a partir de simulações com modelo proposto neste trabalho. Todavia, como é normal neste tipo de aplicação, há algumas limitações que têm de ser consideradas, principalmente para aqueles que venham a trabalhar na extensão dos conceitos e idéias aqui apresentados. Uma das maiores restrições está na perfeita determinação dos parâmetros. Neste trabalho, foi deixado que um ajuste numérico em relação aos dados disponíveis fornecesse o valor dos parâmetros, porém, os valores obtidos devem ser mais bem investigados sob outras perspectivas, que incluam ferramentas de pesquisa mercadológica e econométricas clássicas. Isso dará mais robustez aos resultados obtidos ou então permitirá o surgimento uma crítica compreensiva

O nível de satisfação e familiaridade dos adotantes de uma população pode ser representado em termos do número de anos que a introdução do novo produto tem de atraso em relação à introdução em populações líderes na difusão do produto (TALUKDAR; SUDHIR; AINSLIE, 2002). A lógica é a de que um produto que tenha estado no mercado por vários anos, atendendo uma determinada população, tem mais chances de ter tido seus erros e falhas corrigidos, e de ter desenvolvido uma longa curva de aprendizado com os fabricantes. Logo, adotantes em populações que estão atrasadas na introdução de um determinado produto possuem uma chance maior de ficar inicialmente satisfeitos com os novos produtos do que em países que foram líderes na adoção (GANESH; KUMAR, 1996). Além disso, os consumidores acabam estando mais familiarizados com os benefícios do produto devido ao fato de o produto estar há mais tempo exposto em outros mercados (ROGERS, 1995). Aparentemente é isto o que ocorreu em relação aos telefones celulares pré-pagos no Brasil. Que na sua introdução, passaram a apresentar níveis de penetração significativamente mais altos do que os telefones pós-pagos, introduzidos quase uma década antes.

Outro aspecto que pode ter estimulado esse crescimento éo fato de que a vontade de pagar para adquirir um determinado produto depende basicamente do benefício incremental ofe- dos fenômenos aqui descritos.

Também devem ser considerados outros aspectos econômicos que não foram considerados para construção dos cenários, por exemplo, aqueles referentes às políticas regulatórias do país. Tais aspectos têm impacto sobre o processo de difusão de linhas celulares na medida em que perturbam a oferta. Essa perturbação pode ocorrer por meio da liberação de ofertas complementares ou por meio do bloqueio dessas ofertas. Isso pode elevar o benefício de uso de um produto, por exemplo, devido à escassez e à exclusividade de um número limitado de operadoras e fabricantes.

O uso de modelos de simulação em geral permite aos gestores a definição de formas mais seguras e objetivas para lidar com suas expectativas relativas a projetos que envolvam a difusão de novos produtos. Análises mais estruturadas do processo de difusão de produtos, como a apresentada neste trabalho, poderiam evitar, ou pelo menos ajudar a minimizar, estes e outros tipos de problemas na formulação de estratégias. Este trabalho contribuiu com a construção de um modelo simples que oferece aos acadêmicos, e àqueles que trabalham com planejamento no setor de tecnologia, principalmente de telefonia celular, uma forma eficiente para estudar e entender o processo de difusão desta tecnologia no Brasil. 


\section{Artigo recebido em 09/2007 Aprovado para publicação em 12/2008}

\section{REFERÊNCIAS}

BASS, F. A new product growth model for consumer durables. Management Science, v. 15, n. 5, p. 215-227. 1969

BROWN, L. Innovation Diffusion: A New Perspective. London: Methuen. 1981

DICKERSON, M. D.; GENTRY, J. W. Characteristics of adopters and non-adopters of home computers. Journal of Consumer Research, v. 10, p. 225-235. 1983.

EDEN, C. Cognitive Mapping. European Journal of Operational Research, v. 36, n. 1, p. 1-13, 1988

FAHEY, L.; RANDAL, R. M. Learning from the Future. Nova York: John Wiley \& Sons, 1998.

FORRESTER, J. W. Industrial Dynamics. Cambridge: MIT Press, 1961.

FORRESTER, J. W. Urban Dynamics. Cambridge: Pegasus Communications, 1969.

GANESH, J.; KUMAR, V. Capturing the crossnational learning effect: An analysis of an industrial technology diffusion. Journal of the Academy of Marketing Science, v. 24, p. 328327, 1996.

GEORGANTZAS, N.; ACAR, W. ScenarioDriven Planning: Learning to Manage Strategic Uncertainty. Westport: Quorum Books, 1995.

HAUSER, J.; TELLIS, G. J.; GRIFFIN, A. Research on Innovation and New Products: A review and Agenda for Marketing Science. Marketing Science, v. 25, n. 6, p. 687-717, 2006
HORSKY, D. The effects of income, price and information on the diffusion of new consumer durables. Marketing Science, v. 9, n. 4, p. 342365, 1990.

HORSKY, D.; SIMON, L. S. Advertising and the diffusion of new products. Management Science, v. 1, p. 31-47. 1983

KALISH, S. A new product adoption model with price, advertising and uncertainty. Management Science, v. 31, n. 12, p. 1569-1585, 1985.

KALISH, S.; LILIEN, G. L. A market entry timing model for new technologies. Management Science, v. 32, n. 4, p. 194-205. 1986.

KELLY, G. A. The Psychology of Personal Constructs. Nova York: W. W. Norton, 1955

PIDD, M. Modelagem Empresarial: Ferramentas para a tomada de decisão. Porto Alegre: Bookman, 1998.

POWELL, M. J. D. UOBYQA: unconstrained optimization by quadratic approximation. Report DAMTP 2000/NA14, University of Cambridge. 2000.

ROBERTS, N.; ANDERSEN, D. F.; DEAL, W. A. Introduction to computer simulation: A system Dynamics modeling approach. Massachusetts: Addison-Wesley, 1983.

ROGERS, E. Diffusion of Innovations. Nova York: Free Press, 1995.
SENGE, P. M. A Quinta Disciplina: Estratégias e Ferramentas para Construir uma Organização que Aprende - Caderno de Campo. Rio de Janeiro: Qualitymark, 1994.

STERMAN, J. D. Business Dynamics: System Thinking and Modeling for a Complex World. Boston: McGraw-Hill, 2000.

STONEMAN, P. The Economics of Technological Diffusion. Cambridge, MA: Blackwell, 2002.

TAKADA, H.; JAIN, D. Cross-national analysis of diffusion of consumer durable goods in pacific rim countries. Journal Marketing, v. 55, p. 48-54, 1991.

TALUKDAR, D.; SUDHIR, K.; AINSLIE, A. Investigating New Product Diffusion Across Products and Countries. Marketing Science, v. 21, n. 1, p. 97-114, 2002

VAN DEN BULTE, C.; STREMERSCH, S. Social Contagion and Income Heterogeneity In New Product Diffusion: A Meta-Analytic Test. Marketing Science, v. 23, n. 4, p. 530-544. 2004.

VENSIM. Vensim Help Manual. Vensim 5.5d. Disponível em: <http://www.vensim.com>. Acessado em: 05 set. 2007.

WRIGHT, M.; CHARIETT, D. New product diffusion models in marketing: an assessment of two approaches. Marketing Bulletin, v. 6, p. 32-41, 1995.

\section{SOBRE 0 AUTOR}

\section{Júlio César Bastos de Figueiredo}

Escola Superior de Propaganda e Marketing (ESPM)

End.: Rua Joaquim Távora 1240, 2oandar - São Paulo - SP - 04015-013

Tel.: (11) 5081-8176

E-mail: jfigueiredo@espm.br 